\title{
Safeness of Endoscopic Resection in Patients with End-Stage Renal Disease on Dialysis
}

\author{
Sun-Jin Boo \\ Department of Internal Medicine, Jeju National University School of Medicine, Jeju, Korea
}

See "Bleeding after Endoscopic Resection in Patients with End-Stage Renal Disease on Dialysis: A Multicenter Propensity ScoreMatched Analysis" by In Kyung Yoo, Chan Gyoo Kim, Young Ju Suh, et al., on page 452-457.

Endoscopic resection (ER), by either endoscopic mucosal resection (EMR) or endoscopic submucosal dissection (ESD), is a well-established method as treatment for superficial gastrointestinal tumors. ${ }^{1}$ However, ER may be accompanied by the risk of adverse events such as bleeding or perforation associated with the procedure. In particular, bleeding after ER is one of the most frequent adverse events. In general, post-ER bleeding occurs in about $5 \%$ of cases after the procedure, and ESD is known to have a higher risk of bleeding than EMR. ${ }^{2}$ Many variables including male gender, the size of the tumor and resected specimen, chronic kidney disease (CKD), liver cirrhosis, and the use of antithrombotic drugs are known risk factors of bleeding after ER., ${ }^{2,3}$ Dialysis among patients with CKD is associated with an increased post-ER bleeding risk. Previous studies have reported that the rate of post-ESD bleeding in hemodialysis patients is $20 \%$ to $33 \%$. ${ }^{4,5}$ Various factors in CKD patients lead to decreased platelet aggregation and prolonged bleeding time. Moreover, using anticoagulant agents during dialysis may cause hemostatic abnormalities. ${ }^{6}$ Previous small studies have also reported that gastric acid

Received: July 6, 2020 Revised: July 16, 2020

Accepted: July 16, 2020

Correspondence: Sun-Jin Boo

Department of Internal Medicine, Jeju National University School of Medicine, 15 Aran 13-gil, Jeju 63241 Korea

Tel: +82-64-754-8122, Fax: +82-64-727-3114, E-mail: sunjinboo@jejunu.ac.kr ORCID: https://orcid.org/0000-0002-9945-6766

(c) This is an Open Access article distributed under the terms of the Creative Commons Attribution Non-Commercial License (http://creativecommons.org/ licenses/by-nc/3.0) which permits unrestricted non-commercial use, distribution, and reproduction in any medium, provided the original work is properly cited. hypersecretion and hypergastrinemia exist in some end-stage renal disease (ESRD) patients on hemodialysis. ${ }^{7,8}$

In this issue of Clinical Endoscopy, Yoo et al. ${ }^{9}$ showed that ESRD on dialysis was a significant risk for post-ER bleeding when compared with non-ESRD cases (25.5\% vs. $5.3 \%)$. In both groups of this study, most of the ER methods were ESD, and EMR was performed only in less than $5 \%$ of patients. Although ESRD patients on dialysis had more frequent post-ER bleeding than non-ESRD patients, all cases of bleeding were treated endoscopically without complications. They suggested that endoscopists should not hesitate to endoscopically resect gastric neoplasms in ESRD patients on dialysis due to concerns about bleeding.

There are some things to consider to reduce the risk of bleeding when ER is performed in ESRD patients on dialysis. ESRD patients receiving hemodialysis are at a higher risk of post-ER bleeding than CKD patients who do not require hemodialysis. ${ }^{4}$ The use of anticoagulants such as heparin during hemodialysis may increase the risk of post-ER bleeding. Hemodialysis is desirable to be performed the day before ER, and in hemodialysis session, it is recommended to use nafamostat mesylate as a hemodialysis anticoagulant instead of heparin. ${ }^{4}$ Second-look endoscopy may be more important when ER is performed in ESRD patients on dialysis than in non-ESRD patients. Goto et al. suggested that second-look endoscopy to prevent delayed bleeding after ESD may be omitted to avoid an excessive or unnecessary examination since the rates of delayed bleeding before and after the second-look endoscopy were $2.8 \%$ and $2.5 \%$, respectively. ${ }^{10}$ On the other hand, Numata et al. suggested that second-look endoscopy may be more 
helpful in high-risk patients of post-ESD bleeding and second-look endoscopy should be performed as soon as possible within 24 hours after ESD in hemodialysis patients. ${ }^{4}$

In this study, although the rate of post-ER bleeding was high in ESRD patients on dialysis, all patients who had bleeding were successfully treated endoscopically without sequelae. ${ }^{9}$ However, Numata et al. reported two cases of death due to post-ESD bleeding in ESRD patients undergoing hemodialysis, one caused by femoral infarction, and the other caused by alveolar hemorrhage after post-ESD bleeding. ${ }^{4}$ Because CKD patients have a high risk for cardiovascular diseases, it is important to monitor whether complications occur after post-ER bleeding, even if bleeding is well treated. Although there are difficulties in performing endoscopic treatment for ESRD patients on hemodialysis, it is recommended that ER should be the first therapeutic option for completely resectable tumors, since it is apparent that ER is less invasive than surgery.

This study has limitations of a retrospective research, but it has the strength that many ESRD patients on dialysis were enrolled from 10 centers and propensity score matching was used to reduce the confounding effects of a retrospective study. Additional prospective studies will be needed to verify the safeness of ER in patients with ESRD on dialysis.

\section{Conflicts of Interest}

The author has no financial conflicts of interest.

\section{REFERENCES}

1. Kataoka Y, Tsuji Y, Sakaguchi Y, et al. Bleeding after endoscopic submucosal dissection: risk factors and preventive methods. World J Gastroenterol 2016;22:5927-5935.

2. Libânio D, Costa MN, Pimentel-Nunes P, Dinis-Ribeiro M. Risk factors for bleeding after gastric endoscopic submucosal dissection: a systematic review and meta-analysis. Gastrointest Endosc 2016;84:572-586.

3. So S, Ahn JY, Kim N, et al. Comparison of the effects of antithrombotic therapy on delayed bleeding after gastric endoscopic resection: a propensity score-matched case-control study. Gastrointest Endosc 2019;89:277-285.e2.

4. Numata N, Oka S, Tanaka S, et al. Clinical outcomes of endoscopic submucosal dissection for early gastric cancer in patients with chronic kidney disease. J Gastroenterol Hepatol 2013;28:1632-1637.

5. Matsumura T, Arai M, Maruoka D, et al. Risk factors for early and delayed post-operative bleeding after endoscopic submucosal dissection of gastric neoplasms, including patients with continued use of antithrombotic agents. BMC Gastroenterol 2014;14:172.

6. Rios DR, Carvalho M, Lwaleed BA, Simões e Silva AC, Borges KB, Dusse LM. Hemostatic changes in patients with end stage renal disease undergoing hemodialysis. Clin Chim Acta 2010;411:135-139.

7. Dinoso VP Jr, Murthy SN, Saris AL, et al. Gastric and pancreatic function in patients with end-stage renal disease. J Clin Gastroenterol 1982;4:321-324.

8. Sullivan SN, Tustanoff E, Slaughter DN, Linton AL, Lindsay RM, Watson WC. Hypergastrinemia and gastric acid hypersecretion in uremia. Clin Nephrol 1976;5:25-28.

9. Yoo IK, Kim CG, Suh YJ, et al. Bleeding after endoscopic resection in patients with end-stage renal disease on dialysis: a multicenter propensity score-matched analysis. Clin Endosc 2020;53:452-457.

10. Goto O, Fujishiro M, Kodashima S, et al. A second-look endoscopy after endoscopic submucosal dissection for gastric epithelial neoplasm may be unnecessary: a retrospective analysis of postendoscopic submucosal dissection bleeding. Gastrointest Endosc 2010;71:241-248. 